\title{
¿Por qué Pegan los Niños? Creencias sobre la Agresividad Infantil en un Grupo de Profesoras de Educación Inicial
}

\section{Mercedes Julisa Loza y Susana Frisancho}

Pontificia Universidad Católica del Perú

Mercedes Julisa Loza es Licenciada en Psicología Educacional por la Pontificia Universidad Católica del Perú (PUCP). Licenciada en Educación Inicial por el Instituto Pedagógico Nacional Monterrico. Capacitadora Monitora del Programa Nacional de Formación y Capacitación Permanente Item 02 de la PUCP. Tiene experiencia en el trabajo con infantes y se orienta a la investigación de la relación entre el docente, el clima de aula y los procesos de aprendizaje de los estudiantes.

Susana Frisancho es Ph.D. en Psicología del Desarrollo por la Universidad de Fordham, New York. Magister en Psicología y Psicóloga Educacional por la Pontificia Universidad Católica del Perú (PUCP). Profesora del Departamento de Psicología de la PUCP. Se ha especializado en la relación entre la construcción del conocimiento, el desarrollo humano y la educación, con especial énfasis en el desarrollo moral y cognitivo y la educación moral.

El presente artículo se basa en el trabajo de tesis para optar el grado de Licenciada en Psicología con mención en Psicología Educacional que Mercedes Julisa Loza realizó en los años 2008 - 2009, bajo la asesoría de Susana Frisancho. 


\title{
¿Por qué Pegan los Niños? Creencias sobre la Agresividad Infantil en un Grupo de Profesoras de Educación Inicial
}

\begin{abstract}
Resumen
El presente estudio tuvo como objetivo explorar las creencias que un grupo de profesoras y auxiliares de educación inicial tienen sobre las conductas agresivas que presentan los niños. A través de entrevistas, se exploran las creencias de las docentes en cuatro áreas distintas: a) concepción de agresividad, b) causas probables de la agresividad, c) efectos que tienen los comportamientos agresivos en el aula y d) creencias sobre el manejo de los comportamientos agresivos. Las participantes (10 docentes y 8 auxiliares) constituían el total del personal docente de una institución educativa inicial de gestión pública, de la ciudad de Lima. La investigación muestra que las participantes tienen dificultades para definir el concepto de agresividad y atribuyen las experiencias en casa con la familia el origen de estas conductas, por lo que piensan que son los padres, y no ellas, quienes deben buscar soluciones a dicha problemática. El estudio concluye recomendando crear mayores espacios de reflexión para las docentes a fin de que se sensibilicen sobre su responsabilidad en la formación de los niños en edad preescolar.

Palabras clave: docente de preescolar, agresividad, comportamiento del alumno, educación de la primera infancia
\end{abstract}

\section{Why do Children Hit Others? Kindergarten Teachers' Beliefs about Aggressive Behavior in Children}

\begin{abstract}
This study was aimed at exploring teachers' beliefs about children's aggressive behavior. Through in depth interviews, teachers' beliefs in four different areas were explored: a) their conception of aggressiveness, b) their ideas about its causes, c) the effects that aggressive behaviors have in the classroom dynamics, and d) their beliefs about how these conducts should be managed in the classroom. Participants were 10 teachers and 8 teachers' assistants, who made up the total teachers team of a public early childhood education school in the city of Lima. Results show that participants have difficulties defining the concept of aggression. They also believe that these problematic behaviors stem mainly from home, and so, parents are viewed as the only responsible for the problems and the ones who must find solutions, by themselves. The study ends up by recommending building school opportunities for teachers to exchange ideas and reflect upon their own practice and their responsibility in the education of children in pre-school age.

Keywords: preschool teachers, aggressiveness, student behavior, early childhood education
\end{abstract}




\section{Introducción}

a palabra agresividad deriva de la palabra latina aggressare que significa

aproximarse, atacar. El Diccionario de Lengua Española de la Real Academia Española (RAE), en su vigésima segunda edición (RAE, vigésima segunda edición), define agresividad como la "tendencia a actuar o a responder violentamente". Cierta literatura hace una distinción entre agresividad y violencia, entendiéndose la primera como el comportamiento que causa daño físico o psicológico a otros, y la segunda como un subtipo de comportamiento agresivo que emplea de manera intencional la fuerza física para lastimar a otros, pero en general puede decirse que existe ambigüedad entre ambos términos (Postigo, González, Mateu, Ferrero \& Martorell, 2009).

El comportamiento agresivo de los seres humanos es un tema estudiado desde siempre debido a las enormes implicancias que presenta para la vida en común y a los altos costos que significa para la sociedad en términos del daño material, físico y emocional que causa. Este comportamiento, que tiene tanto determinantes biológicos como ambientales (Bandura, 1986; Bandura, Ross \& Ross, 1961; Raine, 2002), implica una intencionalidad de producir daño a otros, puede estar motivado por la cólera, el dolor, la frustración o el miedo, y algunas veces se manifiesta ya desde la primera infancia. El comportamiento agresivo ha evolucionado como un conjunto de adaptaciones complejas orientadas a lidiar con la competencia en un contexto en el que debemos defendernos, proteger el territorio o las posesiones, obtener recursos para la sobrevivencia o competir por oportunidades reproductivas (Archer, 2009, 1995; Manson \& Wrangham, 1991; Nelson \& Trainor, 2007). La literatura refiere además que la violencia interpersonal, especialmente entre hombres, es un comportamiento presente entre los seres humanos desde épocas remotas (Walker, 2001), y que ciertos patrones son comunes a los humanos y los animales no humanos (Nelson \& Trainor, 2007). Sin embargo, expresados fuera de contexto (como sucede en nuestra época, cuando la mayoría de las funciones adaptativas de la agresión han desaparecido) tanto el comportamiento agresivo como la violencia pueden tener consecuencias indeseables y destructivas (Nelson \& Trainor, 2007).

La psicología ha abordado el problema de la agresión de múltiples maneras. En efecto, los planteamientos para explicar el comportamiento agresivo han ido evolucionando desde teorías tales como la de Lorenz (1976), quien planteó 
que la agresión surge de un instinto de lucha heredado que los seres humanos comparten con otras especies, hasta concepciones más contemporáneas que conciben la agresividad como producto de diversos factores tanto sociales, culturales y situacionales como neuroquímicos y hormonales (Aronson, Wilson \& Akket, 2002; De Rivera, 2003; Discroll, Zinkivskay, Evans \& Campbell, 2006; Dodge, Coie \& Lynam, 2006; Gil-Verona et al., 2002).

Las investigaciones han encontrado que las manifestaciones de la conducta agresiva son distintas a través de las sociedades y difieren de sujeto a sujeto. Cuando las formas de manifestar conductas agresivas es física (golpes, patadas, etc.) suele llamarse agresión directa o agresión instrumental (Card, Stucky, Sawalani \& Little, 2008; Plomin, Nitz \& Rowe, 1990), y se considera más propia de varones. Por el contrario, cuando la expresión de la agresión apunta a dañar las relaciones interpersonales (rechazo, exclusión de un grupo, hablar mal de alguien, etc.), se considera agresión indirecta o agresión relacional (Chaux, 2003; Murray-Close \& Ostrov, 2009; Young, Boye \& Nelson, 2006), la que es practicada con mayor frecuencia por mujeres. La agresión física es la forma más común de agresión en la primera infancia porque requiere menos mediación cognitiva, menos habilidades sociales y menor desarrollo del lenguaje que otras formas de agresión (Coie \& Dodge, 1998; Tremblay, 2004; Tremblay et al., 1999, 2004). La investigación ha demostrado que la presencia de comportamientos de agresión física en la infancia predice problemas de comportamiento violento y no violento durante la adolescencia (Broidy et al., 2003; Nagin \& Tremblay, 1999).

La educación, como proceso social que apunta a propiciar el óptimo desarrollo de las personas, no es ajena a esta problemática. Actualmente, la violencia escolar y la agresividad entre estudiantes es una preocupación mundial; el fenómeno del bullying (acoso o intimidación escolar) -una forma seria de agresión y violencia que tiene efectos duraderos que trascienden la etapa escolar (Furlong, Soliz, Semental \& Greif, 2004; Magendzo \& Toledo, 2008)- ha recibido atención mediática y ha hecho visible para muchas personas la problemática de la agresividad infantil en las escuelas, la que se considera un problema social que debería involucrar tanto a maestros como a psicólogos y otros especialistas en el tema educativo. El bullying es un fenómeno transcultural que ocurre en diversos lugares del mundo (Magendzo \& Toledo, 2008), y si bien es cierto que es más frecuente en los niveles de primaria y secundaria que en los de educación inicial, se sabe que es en la primera infancia cuando los niños empiezan a evidenciar conductas agresivas, 
siendo este el periodo crítico para enseñarles los comportamientos básicos de la interacción social y desarrollar en ellos sus capacidades iniciales de cooperación, convivencia, solución de conflictos y comunicación (Tremblay, Gervais \& Petitclerc, 2008). Además, existe una fuerte correlación entre las conductas delictivas durante la pubertad y los reportes de los maestros sobre el comportamiento de los niños en edad escolar (Roa, Del Barrio \& Carrasco, 2004), y preescolar (Tremblay et al., 2004) por lo que este tema tiene relevancia también para los maestros de educación inicial, especialmente cuando la literatura especializada indica que los comportamientos agresivos se dan con más frecuencia de lo que usualmente pensamos y son una realidad que se observa desde las edades más tempranas (Alink et al., 2006; Keenan, 2009; Tremblay et. al., 2004, 2008; Tremblay, 2000). Como hemos señalado líneas arriba, la agresión física durante la infancia es predictora de problemas comportamentales durante la adolescencia.

Es también relevante señalar que, aunque no es la principal causa de estrés laboral para los docentes, sí es cierto que para muchos maestros las relaciones interpersonales al interior de la escuela, incluyendo la interacción profesor-alumno y la atención a alumnos con problemas de aprendizaje y de comportamiento, constituyen importantes fuentes de tensión (Corvalán, 2005) que minan el trabajo docente efectivo y atentan contra el bienestar de los profesores. Entre otras experiencias docentes que producen malestar (por ejemplo, la poca valoración social de la profesión docente, los bajos salarios, el exceso de carga laboral administrativa, las presiones de tiempo para cumplir con sus funciones o la falta de recursos y materiales) el tener que mantener la disciplina de los niños y lidiar con sus comportamientos inapropiados tiene un lugar como factor generador de estrés (Kyriacou, 2001; UNESCO, 2005). Se ha encontrado además que la percepción que tienen los profesores del mal comportamiento por parte de los niños tiene un considerable efecto indirecto en sus intenciones de abandonar el trabajo docente, y que su eficacia en manejar los problemas de comportamiento infantil media la relación entre la percepción de dicho mal comportamiento y la fatiga mental y desgaste emocional que este les genera (Tsouloupas, Carson, Matthews, Grawitch \& Barber, 2010).

Obviamente, las manifestaciones de agresividad en la infancia deben ser valoradas en función al desarrollo evolutivo de los niños, ya que algunas son propias de la edad y el momento de desarrollo por los que el niño o la niña atraviesa y no representan motivo de alarma ni son señales de patología 
futura. Estudios realizados (Bierman, 2009; Keenan, 2009; Pepler, 2009) evidencian que aproximadamente a partir del primer año los niños muestran algunas conductas agresivas tales como morder, pegar o patear, las que se van incrementando hacia los 2 años (período en el que se presentaría el pico más alto), y se regulan mejor a partir del tercer año de vida debido a la maduración de las capacidades cognitivas y a las estrategias de crianza de los padres. Sin embargo, desconociendo estas características evolutivas, muchas veces los docentes etiquetan a los niños como "agresivos" cuando ante un evento estos simplemente reaccionan con conductas propias de su edad (Roa et al., 2004). Detrás de este juicio se encuentran factores como el nivel de información sobre el desarrollo del niño y las creencias que el docente ha ido construyendo sobre el tema, las que guiarán la forma en que este reaccione a las conductas agresivas de los niños dentro del aula, pues como refiere la literatura especializada, muchas veces son las creencias $-y$ no la información consensuada y objetiva- las que llevan a los docentes a interpretar, decidir y actuar en su práctica educativa (Rodrigo, Rodríguez \& Marrero, 1993).

\section{¿Qué son las Creencias y Cómo se Forman?}

Las creencias son el conjunto de información que una persona tiene acerca de un objeto, son los eslabones cognoscitivos entre el objeto y varios de sus atributos, y se basan no en teorías comprobadas sino en los juicios y evaluaciones subjetivas que el sujeto hace sobre el mundo. Las creencias juegan un papel importante para las personas, pues proveen elementos de estructura, direcciones de orden y valores compartidos. Pajares (1992) afirma que el sistema de creencias reduce las disonancias y confusiones; la gente crece cómodamente con sus creencias y estas a su vez se convierten en su self, de modo que los individuos son identificados y entendidos por la naturaleza de sus creencias y los hábitos que poseen. Las creencias no pueden ser directamente observadas, sino que deben ser deducidas de lo que la gente dice, se propone y hace, pues son ellas las que empujan al individuo a realizar acciones que las confirmen, reforzándolas aunque sean erróneas.

Rodrigo et al. (1993), indican que a nivel del conocimiento las personas hacen uso de las teorías para reconocer o discriminar entre varias ideas, mientras que a nivel de las creencias las personas usan la teoría de modo mucho 
más práctico y valorativo, esto es, centrado en sus intereses, deseos y afectos y no en conocimientos consensuados u objetivos, para interpretar situaciones y realizar inferencias para la comprensión y predicción de sucesos. En este sentido, tal como plantea Pajares (1992), las creencias están basadas no tanto en los conocimientos objetivos que la persona tiene sobre determinado tema u objeto sino en juicios subjetivos, procesos afectivos y valorativos, intereses y gustos y en mecanismos de evaluación.

En general, las creencias se van formando mediante las experiencias de vida y la información que estas experiencias le proveen a la persona, lo que permite la elaboración de esquemas mentales (Perlman, 1985). Para Pajares (1992), mientras más temprano una creencia es incorporada en la estructura de creencias del sujeto, más difícil es alterarla; así, las creencias subsecuentemente afectan las percepciones e influyen fuertemente en el procesamiento de nueva información. Esto explica por qué con el tiempo y el uso las creencias se vuelven más fuertes y los individuos pueden mantenerlas aun cuando hayan recibido explicaciones que las contradigan.

Creencias docentes. Las creencias de los profesores pueden y deben convertirse en un tema importante de investigación, pues su práctica docente y el manejo de aula están estrechamente ligados a ellas. Como afirma Kagan (1992), las creencias están en el corazón mismo de la enseñanza.

Todos los profesores tienen creencias sobre el mundo en general y sobre la educación en particular, que funcionan como concepciones asumidas como verdaderas (Clark, 1988, citado por Pajares, 1992), las que los orientan a interpretar, planear y tomar decisiones que afectan la acción educativa. Las creencias están presentes incluso antes de haber iniciado la formación profesional, pues como se ha mencionado, los individuos van construyendo su sistema de creencias a partir de sus experiencias de vida, desde temprana edad. No obstante, las creencias de los docentes al iniciar su formación profesional se ven afectadas de manera directa por la interpretación y valoración que estos hacen de las nuevas experiencias y de sus procesos de formación como profesores (Marcelo, 2005). Leal (2005) afirma que las experiencias que los docentes tuvieron como estudiantes ya los han conducido a desarrollar ciertas creencias acerca del proceso educativo y el conocimiento, las que hacen que el adquirir nuevos conocimientos o modificar los que ya tienen pueda resultar difícil, conflictivo o amenazante según como se haya articulado su sistema de creencias pues, como se sabe, no todas las creencias tienen el mismo peso o la misma 
fuerza en un mismo sujeto. En efecto, diversos autores han planteado que las creencias se organizan en un sistema en el que existen creencias centrales y periféricas; como su nombre lo indica, las primeras son centrales y prevalecen sobre las segundas cuando se filtra información, se evalúa y se toman decisiones (Haney \& Mcarthur, 2002; Pajares, 1992; Rokeach, 1968). La investigación ha mostrado que el campo de las creencias docentes es complejo, ya que estas pueden no caer limpiamente en categorías discretas (Olafson \& Schraw, 2006) pudiendo los docentes tener al mismo tiempo creencias que son a la vez generales y específicas. Además, las creencias pueden ser independientes unas de otras, posibilitando la existencia, en la misma persona, de creencias contradictorias. Esto explicaría por qué muchos docentes a nivel discursivo manifiestan una concepción determinada de las cosas pero a nivel práctico hacen exactamente lo contrario (Ministerio de Educación del Perú, 2006) sin que parezcan ser conscientes de la contradicción.

Existen múltiples estudios que parten de identificar las creencias docentes, creencias epistemológicas o creencias pedagógicas por ejemplo, para luego intervenir sobre ellas y mejorar la práctica educativa (Buehl, 2009; Leal, 2005; Kardash \& Howell, 2000; Macotela, Flores \& Seda, 2001; Pajares, 1992), pues existe evidencia de que no es posible el cambio educativo si el profesor no cambia también sus creencias sobre diversos temas, especialmente acerca de cómo se aprende y cómo se enseña (Gómez, Santa Cruz \& Thomsen, 2007). En relación a la agresividad infantil y las creencias que los profesores tienen sobre el tema, los docentes suelen identificar ciertos comportamientos infantiles como masculinos o femeninos, y diferencian con claridad la agresión física de otras formas de agresión (Spilt, Koomen, Thijs, Stoel \& Van der Leij, 2010). Se sabe también que los docentes creen que para asegurar su éxito académico es fundamental el modo que tengan de manejar las conductas agresivas en los niños (Vitaro, Brendgen, Larose \& Tremblay, 2005). Debido a que el profesor concibe el éxito académico como estrechamente ligado a la disciplina escolar, la que tendría la meta de aumentar los minutos dedicados al trabajo efectivo (Woollfolk, Davis \& Pape, 2006), el manejo que haga el profesor de aula de los alumnos que presenten conductas agresivas es percibido como fundamental. Dicho manejo dependerá, según Woolfok et al. (2006), de la manera en que los profesores definieron y conciben su papel en el salón de clase.

Dado que las creencias que las personas tienen sobre el mundo influyen en la manera en que se relacionan con él y actúan sobre él (Pajares, 1992), que los 
comportamientos agresivos de los niños en la escuela son una realidad que no se puede soslayar, y que las creencias docentes están a la base de lo que las profesoras hacen o no en las aulas (en este caso, su manera de afrontar el problema de la agresividad infantil y la responsabilidad que se atribuyen al respecto), en este estudio nos propusimos responder a la siguiente pregunta: ¿cuáles son las creencias que un grupo de docentes y auxiliares de educación inicial tiene sobre el comportamiento agresivo de los niños? Para responder a esta pregunta general, establecimos como objetivos específicos los siguientes cuatro: 1) explorar las creencias de las docentes sobre el concepto de agresividad, 2) explorar sus creencias sobre las causas de la agresividad de los niños, 3) explorar sus creencias sobre los efectos que la agresividad de los niños tiene en el aula y 4) explorar las creencias docentes sobre los modos de manejar estas conductas.

Si bien se trata de un estudio de caso que explora lo que ocurre con un grupo de profesores de una escuela de educación inicial en particular, y por esa razón no es en esencia generalizable desde los criterios de un enfoque cuantitativo que demanda muestras estadísticamente representativas, consideramos que al tratarse de una institución pública similar a muchas otras existentes en el país, esta primera aproximación puede darnos luces sobre lo que podría ocurrir también en otras instituciones educativas que comparten sus características (ver Eisner, 2003). Además, dado que la mayor parte de referencias teóricas y empíricas son del extranjero, este trabajo ayuda a enfocar el tema en el Perú, donde lamentablemente contamos con muy escasa investigación sobre el mismo.

\section{Metodología}

\section{Participantes}

La selección de participantes fue intencional. Participaron 10 profesoras de Educación Inicial cuyas edades fluctúan entre los 30 y 50 años, y 8 auxiliares de educación con edades entre los 28 y 50 años, de una institución educativa inicial pública del distrito de Los Olivos, en la ciudad de Lima. Cabe resaltar que estas personas constituyen el total de la población de docentes y auxiliares del colegio. Todas las participantes, tanto docentes como auxiliares, firmaron un consentimiento informado sobre las condiciones de su inclusión en este estudio y aceptaron voluntariamente participar en él. 
El 100\% del personal docente tiene estudios acordes con el nivel educativo en el que se viene desempeñando. Este no es el caso de las auxiliares, ya que sólo el $25 \%$ de ellas indica tener estudios concluidos en educación inicial. Respecto a su formación profesional se aprecia que el $50 \%$ de las docentes ha cursado estudios universitarios mientras que el otro $50 \%$ lo ha hecho en institutos superiores, tanto de gestión pública como de gestión privada. En el caso de las auxiliares de educación la distribución indica que el mayor porcentaje (5 docentes, 63 \% de la muestra) ha cursado estudios en institutos superiores. Respecto al tiempo de experiencia laboral de las participantes la distribución es la siguiente:

\section{Tabla 1}

Años de Experiencia Laboral de las Participantes

\begin{tabular}{lccc}
\hline Tiempo de servicio & Docentes $\mathrm{N}$ & Auxiliares $\mathrm{N}$ & Total N \\
\hline De $6-11$ años & 3 & 3 & 6 \\
De $12-17$ años & 3 & 1 & 4 \\
De $18-23$ años & 4 & 4 & 8 \\
Total & 10 & 8 & 18 \\
\hline
\end{tabular}

\section{Medidas}

El estudio se realizó con dos medidas, una Ficha de Datos Demográficos y una Entrevista Semi-estructurada, diseñadas ambas para esta investigación.

Ficha de Datos Demográficos. Apunta a recoger datos de filiación tales como los años de estudios, el tipo de centro de formación inicial y la experiencia laboral, a fin de conocer y caracterizar a las participantes.

Entrevista. Orientada a indagar las creencias de los docentes acerca de la agresividad de los niños. La revisión bibliográfica (Dodge et. al., 2006; Baron \& Byrne, 2005; Aronson et al., 2002; Cerezo, 1997; Lorenz, 1976; Bandura et al., 1961) indica que tanto la concepción de la agresividad como sus causas han sido tema de extensos estudios, por lo que éstas fueron dos áreas que se incluyeron en la entrevista. Por otro lado, la literatura también señala como importante el clima del aula y el manejo que el docente tenga de los comportamientos agresivos de los niños en edad preescolar (Calderhead, 1996; Woolfolk, 1999). Por ello estos dos aspectos conformaron las otras dos áreas de la entrevista, por lo que el instrumento final contó con cuatro áreas de exploración: 
Área 1: creencias sobre el concepto de agresividad. Son todas aquellas ideas que los docentes tienen acerca del concepto de agresividad y de las conductas que se consideran agresivas, es decir, su maneras de entender la agresividad, de conceptualizarla y establecer diferencias con otros conceptos similares, así como los modos en que reconocen (o no) conductas que podrían clasificarse como agresivas.

Son ejemplos de preguntas de esta área las siguientes:

- ¿Qué es para usted la agresividad? ¿Podría dar algunos ejemplos de comportamientos agresivos?

- Desde su punto de vista, ¿̇qué comportamientos muestran los niños y niñas agresivos? ¿Todos los niños que se muestran agresivos presentan las mismas conductas, o hay diferencias?

Área 2: creencias sobre las causas de la agresividad. Son las creencias de los docentes referidas a las causas de las conductas agresivas de los niños, sus ideas acerca de qué procesos o factores podrían subyacer y/o explicar el comportamiento agresivo infantil.

Dos ejemplos de preguntas de esta área son los siguientes:

- ¿Por qué cree usted que un niño o niña muestra conductas agresivas? ¿Qué lo hace agresivo?

- ¿Cuáles cree usted que serían las causas de la conducta agresiva? En su experiencia, ¿¿cuál sería la causa principal?

Área 3: creencias sobre las consecuencias de la agresividad. Son aquellas ideas que los docentes han construido en relación a cómo las conductas agresivas influyen en la dinámica del aula y en las relaciones interpersonales en la escuela.

Ejemplos de este tipo de preguntas son los siguientes:

- Para usted, ¿̇cómo influye la agresividad de los niños en la dinámica de grupo del aula?

- En relación al rendimiento, ¿̇cree usted que los niños con comportamientos agresivos rinden más, igual o menos que los que no presentan conductas agresivas? ¿Por qué?

Área 4: creencias sobre el manejo de estas conductas. Esta área se refiere a las ideas que los docentes tienen acerca de cuáles son las maneras más eficaces de manejar las conductas agresivas en el aula. 
A continuación presentamos algunas preguntas de esta área:

- ¿Qué cree usted que un docente/auxiliar debe hacer frente a un niño agresivo? ¿Por qué?

- Cuando ha tenido un niño con conductas agresivas en el aula, ¿qué medidas ha tomado usted? ¿Cuáles han sido las más eficaces? ¿Por qué?

Procedimiento. La entrevista se construyó a partir de la revisión teórica de la literatura especializada, la que sirvió para la identificación de las cuatro áreas que se incluyen en la misma. La primera propuesta de entrevista, elaborada en función de la revisión bibliográfica y los fines de la investigación, pasó por cuatro jueces expertos que la analizaron y emitieron juicio acerca de su estructura, la pertinencia de sus áreas y la calidad de las preguntas. El formato de la entrevista que se les alcanzó a los jueces contenía las preguntas distribuidas en desorden, de tal manera que ellos debieron indicar a qué área de la entrevista pensaban que correspondía cada pregunta. Las preguntas se afinaron según las indicaciones dadas por los jueces y se eliminaron aquellas en los que dos jueces o más encontraban deficiencias.

En la generalidad de los casos la entrevista se realizó en una sola sesión, en los propios ambientes de la institución educativa. Todas las entrevistas fueron grabadas y transcritas posteriormente. Las respuestas dadas por las docentes fueron agrupadas en categorías que se construyeron en base al análisis de contenido de las propias respuestas (no se establecieron categorías a priori); dichas categorías fueron elaboradas por la investigadora principal y contrastadas luego con la asesora que actuó como juez independiente. De esta manera se afinaron las categorías y se discutieron los criterios para las mismas hasta lograr el acuerdo respecto a su pertinencia y sentido. Respecto al proceso de entrevista, es importante señalar que a las docentes y auxiliares se les especificó las condiciones de su participación, dejando ellas constancia de su aceptación a participar de manera voluntaria mediante la lectura y firma de un consentimiento informado.

\section{Resultados}

Para una mejor lectura, organizamos los resultados siguiendo las áreas que se exploran en la entrevista. No se hace la distinción entre docentes y auxiliares porque los resultados fueron semejantes para ambos grupos. 


\section{El Concepto de Agresividad}

El análisis de las respuestas a la entrevista nos indica que en general, las docentes identifican las consecuencias negativas que genera el comportamiento agresivo en las demás personas, pero no se aprecia que tengan una definición clara de lo que es la agresividad. Las respuestas muestran que, tal como refiere la literatura (Postigo et al., 2009), existe ambigüedad en los términos empleados por las docentes, que usan como equivalentes agresividad y violencia, sin hacer distinciones. Aunque en la literatura se hace una diferencia entre ambos conceptos, entendiéndose la agresividad como el comportamiento por el que se causa daño físico o psicológico a otros, y la violencia como el comportamiento agresivo que emplea de manera intencional la fuerza física para lastimar a otros (y en este sentido, todo comportamiento violento es agresivo, pero no todo comportamiento agresivo es violento) (Goetz, 2010), no se ha encontrado que las docentes se planteen estas distinciones, sin que haya diferencias en sus respuestas según el tipo de institución educativa de origen, ni el régimen (público o privado), ni los años de estudio, ni la experiencia laboral.

El 67\% (12 participantes) define la agresividad como conductas negativas, reacciones que tiene una persona frente a otra, comportamientos violentos, maltratos físicos o desfogue de emociones. Por ejemplo:

La agresividad viene a ser las conductas que adquieren los niños a través de las vivencias que tienen, son conductas negativas que influyen en ellos... que tienen en su hogar o con las personas cercanas. Las conductas negativas me refiero al maltrato físico o psicológico que puedan tener en su hogar cuando el padre de familia no puede manejar situaciones y lo que hace es agredir ya sea a su esposa o a las personas que están en su entorno. Cuando el niño pega a sus compañeros los golpea, de repente las cosas las empieza a malograr a través de los golpes o movimientos toscos que pueden tener. (Docente 3, 37 años).

Comportamientos violentos de los niños... manifestaciones en forma violenta, aparatosa, para conversar, en su expresión, en su conducta, en su forma de ser, para hablar, para tratar a sus compañeros... La manifiestan pegando a sus compañeros, golpeando la mesa, la silla, maltratando a sus compañeros. Por ejemplo cuando un niño está jugando y golpea sus compañeros no los respetan, están trabajando y le rayan la hoja, o no los dejan jugar. (Docente 6, 41 años). 
La agresividad es cuando el niño presenta la conducta de pelearse, de pegar. Es cuando un niño sin razón lastima a otro niño. (Docente 18, 40 años).

Es cuando en clases el niño tira las cosas, jala el pelo, pellizca o muerde, no pide permiso. (Docente 7, 40 años).

Por lo general, las participantes definen el comportamiento agresivo solamente describiendo sus manifestaciones observables, sin mayor precisión o diferenciación entre dichos comportamientos y sin hacer alusión a otros tipos posibles de conductas agresivas más sutiles o menos observables. Todas coinciden en que los niños que presentan estas conductas causan daño a otros niños o a las demás personas, aunque esta intencionalidad de causar daño no es incluida como parte de la definición, la que aparece muy general y poco precisa. Por otro lado, las docentes refieren que las manifestaciones de la agresividad son diferentes de niño a niño, reconociendo las idiosincrasias que pueden existir en este comportamiento entre las personas. Además, el $100 \%$ coincide en que las expresiones de la agresividad se manifiestan de diferentes maneras tanto en los niños como en las niñas y el $76 \%$ afirmó explícitamente que existen diferencias de género en la expresión de la agresividad, lo que va de acuerdo con lo que indican las investigaciones revisadas. Por ejemplo:

Me parece que los niños son un poquito más agresivos que las niñas. Esto sucede porque los niños ven programas de violencia, programas donde se pelean y vienen al colegio y quieren hacer lo mismo, en cambio en las niñas no. (Docente 16, 39 años).

El varón es más violento, las niñas son más cohibidas. Los varones pelean más provocan a sus compañeros, a veces que se quitan las loncheras. (Docente 11, 50 años).

Por ejemplo los niños presentan la agresividad golpeando a sus compañeros ya sea niño o niña, de igual manera los golpean ellos. En cambio las niñas demuestran su agresividad quitando algo que ellas han traído por ejemplo una muñeca que es un juguete. (Docente 5, 46 años).

Estas diferencias las explican por el trato que reciben los niños de parte de sus padres, pues mientras que a ellos se les incita a juegos más rudos y a 
defenderse por sí mismos, las docentes piensan que a las niñas se les tiende a sobreproteger.

\section{Las Causas de la Agresividad}

Son dos las posibles causas de la agresividad que las profesoras identifican: las características del ambiente familiar o social (amistades, televisión, vecinos) y los aspectos biológicos ó genéticos. Es el entorno familiar el lugar principalmente señalado como fuente de observación de estos comportamientos por parte de los niños (14 profesoras, $77 \%$ de participantes), asumiéndose una postura de aprendizaje directo de la agresividad a través de la observación de comportamientos agresivos en sus familias:

La familia... la causa principal sería que el niño esté observando el maltrato de los padres... de que los padres castiguen a los niños de manera física y verbal. (Docente 2, 30 años).

Principalmente el niño es el reflejo de los padres de lo que ven en casa, si bien es cierto el niño pasa buen tiempo en el colegio, pero todo lo trae de casa lo que ha aprendido, lo bueno y lo malo, aquí corriges ciertas cosas pero todo para mi se inicia en el hogar, como ha sido tratado como ha sido educado... que él vea que dentro de su casa de repente hay agresión eso se refleja en su conducta, el entorno familiar. (Docente 14, 37 años).

Como causas biológicas se identifican las condiciones difíciles de concepción o del periodo de gestación, que los padres durante la concepción hayan estado bajo los efectos de alcohol u otras drogas, o que en el embarazo haya habido problemas en la pareja o situaciones difíciles para la madre. Por ejemplo:

Pienso que la agresividad viene cuando los padres engendran ebrios a sus niños, yo he visto muchos casos cuando sus padres eran alcohólicos, pienso que el alcohol influye mucho en el cerebro en algún nervio en el cerebro, en el hipotálamo. De padres alcohólicos los niños nacen agresivos, en mi larga experiencia siempre ha coincidido con los casos que he visto... no sé si es coincidencia pero yo siempre he visto esos casos. (Docente 1, 48 años). 
Otra causa sería el sufrimiento fetal, es decir cuando la madre está gestando por múltiples razones no ha tenido un embarazo adecuado, no ha sido tranquilo, ha sido perturbado por problemas con el padre, ofamiliares en general, no tener dinero, en este caso el feto todo lo va asumiendo. (Docente 4, 50 años).

En la tabla siguiente se ve la variedad de explicaciones que dieron las docentes (varias docentes dieron más de una respuesta por lo que el $\mathrm{N}$ total es mayor que 18):

\section{Tabla 2}

Causas de la Conducta Agresiva

\begin{tabular}{lcc}
\hline Causas & $\mathrm{N}$ & $\%$ \\
\hline $\begin{array}{l}\text { Entorno familiar (problemas familiares, agresión de los padres, } \\
\text { falta de límites en casa) }\end{array}$ & 14 & $77 \%$ \\
$\begin{array}{l}\text { Influencia ambiental más allá de la familia (imitación de } \\
\text { conductas observadas en TV, el colegio o el barrio) }\end{array}$ & 4 & $22 \%$ \\
$\begin{array}{l}\text { Necesidad de llamar la atención por falta de cariño } \\
\begin{array}{l}\text { Causas biológicas (haber sido concebido bajo los efectos del } \\
\text { alcohol, problemas durante la gestación) }\end{array}\end{array}$ & 3 & $16 \%$ \\
\hline
\end{tabular}

Como se aprecia, las razones que tanto docentes como auxiliares ofrecen para explicar las causas del comportamiento agresivo de los niños son diversas y algunas incluso opuestas. Al parecer no existe una teoría sólida acerca de las causas de este tipo de comportamiento sino solamente ciertas hipótesis que funcionan a nivel de creencias pues no parecen estar organizadas de manera lógica.

\section{La Influencia de la Agresividad en el Aula}

Para las docentes, las conductas agresivas son un problema cuando se manifiestan continuamente y perturban el desempeño de las actividades en el aula (10 docentes, 56\%). En segundo lugar ( 8 docentes, $44 \%$ ), el comportamiento 
agresivo es percibido como un problema por las consecuencias que podría generar tanto a nivel de daño a sus compañeros como de perjuicio para el entorno, sobre todo cuando estas consecuencias son graves. Por ejemplo, una docente explica que el comportamiento agresivo de los niños es un problema:

Cuando no se puede controlar, todo para el niño es golpe, patadas, cuando tira las cosas, cuando golpea, cuando rompe, por ejemplo está leyendo un cuento y lo rompe, o agarra algo y lo quiere destruir, está buscando qué destruir, qué jalar, garabatear, quieren garabatear el piso, parten el lápiz y lo tiran. Cuando son agresivos ellos rompen el lápiz o lo tajan, tajan y lo tiran. (Docente 6, 41 años).

En cuanto a cómo influye esta conducta en el grupo de niños, el 89\% (16 docentes) coincide en señalar que los comportamientos agresivos generan distracción, alboroto y desorden en el aula, lo que impide continuar con las actividades programadas:

Tampoco se puede avanzar haciendo una actividad sabiendo que hay ese problema, si se va a hacer una actividad, tiene que ser una actividad con armonía de los niños y si hay un niño que está golpeando, está mordiendo es bien difícil, entonces mejor parar, hablar con él y si es posible que los niños observen la reconciliación que en clase se pueda dar. (Docente 14, 37 años).

Alborota a los niños pero hay que controlar a ese niño, si no te mueve a todo el salón, distorsiona el ambiente, el niño que es agresivo distorsiona el ambiente, es decir el grupo está tranquilo y el niño agresivo hace que se alborote el grupo en ese caso se tiene que poner reglas fuertes para que ese niño cambie, hay que hablarle. (Docente 6, 41 años).

En general, tanto docentes como auxiliares coinciden en que los comportamientos agresivos son distractores e influyen de modo negativo en el clima del aula.

\section{El Manejo de las Conductas}

En relación a las acciones que las docentes creen que se deberían tomar y las que ellas mismas toman para enfrentar las conductas agresivas de los niños, 
el $30 \%$ de participantes afirma que la mejor alternativa radica en conversar con los padres de familia para indagar los motivos que expliquen el comportamiento del niño en clase:

Principalmente hablar con los padres para ver el origen de la agresividad, procurar que sean sinceros para poder ayudarlos de lo contrario no se puede hacer nada. (Docente 4, 50 años).

La docente primero que nada debe conversar bastante con ambos padres, para entender lo que sucede en el hogar y ayudar al niño. (Docente 7, 40 años).

Seguidamente indican que se debería conversar con el niño y buscar ayuda profesional ( $22 \%$ en ambos casos), principalmente de una psicóloga especializada. El 19\% cree que hay que buscar estrategias dentro del aula, por ejemplo establecer normas de conducta claras, tener ocupado al niño, buscar algo agradable para distraerlo o realizar dramatizaciones para abordar el tema con los alumnos. Algunas docentes (7\%) también tienen la creencia de que pueden ayudar al niño brindándole afecto, pues asumen que la necesidad de afecto está a la base de sus problemas de conducta.

Ante la pregunta sobre cuáles son las acciones inmediatas que tomaban ellas en el momento del comportamiento agresivo de un niño, se encuentra que la mayoría de docentes manifiesta dialogar con el niño y hacer que pida disculpas al compañero que agredió (42\% de participantes); asimismo, está la acción de hablar con los padres sobre la conducta del niño (21\%). Como estrategias también utilizan el darle responsabilidades al niño (13\%), buscar un especialista (8\%), dejar que el niño se desfogue y/o dejarlo sin recreo (4\% en cada caso).

Como puede apreciarse, las respuestas de las docentes muestran por un lado el "deber ser", lo cual es distinto a lo que hacen efectivamente cuando deben enfrentar un comportamiento agresivo.

\section{Discusión}

El comportamiento agresivo en las aulas de clase se presenta desde los primeros niveles de escolaridad; como se ha visto, también se da de manera natural en la primera infancia (Keenan, 2009; Tremblay et al., 2008). En esta investigación hemos encontrado que las docentes han presentado considerable 
dificultad para definir este concepto y diferenciarlo de otros afines. Muchas docentes incluyen el término violencia como parte de su definición y toman los términos agresividad y violencia como sinónimos. Malvaceda (2009) afirma que es frecuente la confusión entre estos términos debido a las características de sus manifestaciones; por su parte Ortega y Mora-Merchán (2000) sostienen que ambos conceptos son ambiguos y que es el contexto cultural lo que definirá a cada uno, mientras que Del Barrio (2003, citado en Postigo et al., 2009) plantea que un acto es agresivo por sus efectos y por su intención, y que la violencia se reconoce más por las consecuencias que por sus antecedentes, por lo que existen agresiones violentas y no violentas. Es evidente que esta ambigüedad terminológica y conceptual existente incluso en la literatura científica, ayuda a entender la dificultad de las docentes al definir la agresividad. Sin embargo, aun cuando no tienen un concepto claro y preciso del término agresividad, sí reconocen las manifestaciones de esta conducta, especialmente cuando la agresión es directa (por ejemplo, dar puñetes, pegar, arañar, jalar el cabello, morder) (Baron \& Byrne, 2005) y en segundo lugar cuando es indirecta o relacional (inducir a no juntarse con alguna persona en particular o hablar mal de otras personas) (Chaux, 2003). Cabe preguntarse si la poca claridad conceptual que presentan los docentes respecto a este tema estaría influyendo en que no reconozcan tan fácilmente como agresión ciertas conductas pasivas, poco llamativas y no violentas, tales como las que se presentan en casos de agresión indirecta. De hecho, la literatura reporta que en la generalidad de los casos el bullying no es percibido como tal por los maestros de las escuelas en las que sucede (Magendzo \& Toledo, 2008; Furlong et al., 2004), lo que en parte se debe a que la agresión indirecta, al ser más sutil y menos observable resulta difícil de reconocer.

En relación a las posibles causas de la agresividad, las investigaciones realizadas indican que no hay un solo motivo para que un individuo manifieste conductas agresivas; las docentes señalan también que hay más de una causa para las conductas agresivas de los niños, lo que coincide con los estudios realizados a la fecha (Baron \& Byrne, 2005). Es la familia sin embargo, y las experiencias que el niño vive en ese contexto particular, la razón que priorizan como causa probable de la agresividad. La creencia común de las participantes es que "esos comportamientos vienen de casa". Esto va de la mano con las investigaciones realizadas (Sanders \& Becker, 1995, citado en Almeida, Goncalvez \& Sani, 2009; Underwood, Beron, Gentsh, Galperin \& Risser, 2008) que han reportado que una de las fuentes principales de adquisición del 
comportamiento violento y agresivo de los niños es la familia, especialmente cuando dentro de la misma hay maltrato, lo que funcionaría como modelo para el aprendizaje por observación (Bandura, 1986). En esta línea, los estudios de Goncalves (2003, citado en Almeida et al., 2009) evidencian que la violencia en la familia está directamente relacionada con los comportamientos agresivos que los niños podrían desarrollar más tarde en la escuela. En este sentido, la percepción de las docentes sobre la importancia de la familia no resulta infundada. Sin embargo, el que hayan identificado a la familia como principal causa de los comportamientos agresivos de los niños podría llevarlas a dejar de lado la propia responsabilidad de buscar alternativas de solución al asumir que el problema es estrictamente del hogar, y que es en ese contexto -y no en la escuela- donde se deberían tomar las medidas correctivas o de prevención. Lo mismo puede decirse de su identificación de factores biológicos, usualmente entendidos como permanentes e inmodificables, como causas de la agresividad infantil. Dado que las creencias son fundamentalmente un tipo de comprensión e interpretación con la que el docente representa las situaciones cotidianas, y que estas influyen directamente en lo que el profesor hace en el aula, cuando los docentes piensan que ellos no son parte de la causa del problema o creen que tienen pocas posibilidades de ayudar a revertirlo (porque las raíces del mismo se encuentran fuera de su alcance, en contextos en los que ellos no tienen capacidad de intervención) difícilmente asumirán un rol agente y responsable para su solución.

En relación a la influencia de la agresividad en el aula, cabe preguntarse por qué se ha relativizado tanto la percepción de la agresividad al punto de considerarla un problema solo cuando es muy persistente o tiene consecuencias potencialmente fatales. Al parecer, el umbral de tolerancia a la agresividad es alto, lo que podría deberse a un efecto de desensibilización (Baron, 1974, citado en Baron \& Byrne, 2005), es decir, a la exposición continua a la violencia tanto en la vida diaria como en los medios de comunicación, lo que ha originado que las personas se vuelvan poco o nada sensibles al dolor y al sufrimiento de otros y tengan una menor reacción emocional al observar conductas violentas o agresivas. Esto explicaría que las docentes reaccionen sólo cuando las consecuencias son mayores; antes de eso, las conductas agresivas son incorporadas como actos comunes o comportamientos normales, no siendo percibidos como problema. Sería interesante explorar esta hipótesis en una investigación futura.

Acerca de las estrategias para el manejo de las conductas problemáticas en aula, es importante señalar que las docentes refieren una variedad de ellas, 
desde algunas muy generales y vagas como "darle amor a los niños" hasta otras más precisas como otorgarles ciertas responsabilidades dentro del aula. Si bien, por supuesto, es positivo que las docentes cuenten con diversas alternativas de intervención, también es importante señalar que las mencionadas no son necesariamente las más efectivas, ya que el "mantenerlo ocupado" distraerá al niño pero no le dará alternativas comportamentales que le permitan regularse y modificar su comportamiento. En efecto, investigaciones (Almeida et al., 2009; Aronson et al., 2002; Baron \& Byrne, 2005; Chaux, 2003; Furlog et al., 2004) proponen abordar las conductas agresivas desde dos aspectos básicos: el contexto escolar, donde se puede trabajar programas educativos tales como el desarrollo de habilidades sociales (comunicación, entrenamiento de la empatía entre los alumnos, aprender a identificar emociones y manejarlas constructivamente, etc.), liderazgo, trabajo cooperativo; y el contexto social más amplio mediante la exposición a buenos modelos sociales y reglas claras para castigar las conductas agresivas. Distraerlo resulta ser simplemente una estrategia de corto plazo y no una solución efectiva al problema.

\section{A Modo de Conclusión}

En el sistema educativo, los docentes juegan un papel fundamental debido a su responsabilidad en la planificación, organización, ejecución y evaluación de las actividades educativas propuestas a los niños. Actualmente se ha puesto la mirada sobre el desempeño del docente en el aula, pues en nuestro país, como en muchos otros, la educación pasa por los momentos más críticos de las últimas décadas (Cerdá, 2004), por lo que las reformas educativas que se propongan deben tener en cuenta tanto al alumno como al docente. En este sentido, conocer las creencias de los docentes sobre distintos temas vinculados al ejercicio pedagógico resulta relevante, ya que son ellas las que los llevan a interpretar, decidir y actuar en su práctica educativa (Rodrigo et al., 1993).

Si se asume que las profesoras encargadas de la educación inicial deben estar preparadas para propiciar condiciones adecuadas para la socialización y el desarrollo de los niños, puede concluirse que la información que posean sobre el desarrollo del niño y las creencias que hayan construido acerca de la agresividad infantil son relevantes para su ejercicio pedagógico. Por período evolutivo, así como por las características de la transición hogar- 
escuela por la que atraviesan los niños que inician su escolaridad, se espera que la docente del nivel inicial se encuentre más de una vez con niños que presentan conductas agresivas. ¿Cómo enfrenta esta situación? ¿De qué manera responde a ella? Pensamos que la actividad cotidiana del docente en el aula no debe ser un procedimiento meramente rutinario centrado en lo estrictamente curricular, sino una labor reflexiva que implica ser consciente de las creencias, conocimientos y capacidades que usará en cada situación específica y que orientarán los objetivos de su acción. En este sentido, y de acuerdo a los resultados de este estudio, consideramos necesario que en la formación de docentes se den mayores herramientas acerca del desarrollo del niño en general y sobre el comportamiento agresivo en la infancia en particular, y que en las instituciones educativas se establezcan espacios de reflexión que permitan contrastar las propias creencias y las de los demás, analizarlas críticamente y tomar conciencia de su impacto en la práctica pedagógica. Como se ha visto en este estudio, varias de las creencias que las profesoras y auxiliares que participaron en él tienen sobre la conducta agresiva de los niños podrían atentar contra su participación en la búsqueda de alternativas de solución a esta problemática, lo que atenta a su vez, de manera más amplia y general, contra su rol como agentes educativos reflexivos, innovadores y trasformadores.

Para concluir queremos señalar que este es un estudio inicial y por lo tanto limitado. Quedan como líneas de acción futura ahondar en este tema de investigación explorando con mayor profundidad las creencias docentes acerca de la agresividad infantil e incorporando a otros grupos de profesores, de contextos y niveles educativos distintos, a fin de tener una idea más cabal y completa de este tema de interés educativo. 


\section{Referencias}

Alink, L., Mesman, J., van Zejil, J., Stolk, M., Juffer, F., Koot, H., ... van ljzendoorn, M. (2006). The early childhood aggression curve: Development of physical aggression in 10-to 50-month-old children. Child Development, 77(4), 954-966.

Almeida, T., Goncalvez, R. \& Sani, A. (2009). La agresividad en niños que testimonian la violencia de género. Anuario de Psicología Jurídica, 18, 113-118.

Archer, J. (1995). What can ethology offer the psychological study of human aggression? Aggressive Behavior, 21, 243-255.

Archer, J. (2009). The nature of human aggression. International Journal of Law and Psychiatry, 32(4), 202-208.

Aronson, E., Wilson, T. \& Akket, R. (2002). Social Psychology. New Jersey: Prentice Hall.

Bandura, A. (1986). Social foundations of thought and action: A social cognitive theory. Englewood Cliffs, N. J.: Prentice Hall.

Bandura, A., Ross, D. \& Ross, S. (1961). Transmission of aggression through imitation of aggressive models. Journal of Abnormal and Social Psychology, 63, 575-582.

Baron, R. \& Byrne, D. (2005). Psicología Social. Madrid: Pearson Education.

Bierman, K. (2009). Programas y servicios que han comprobado su efectividad para reducir la agresión en niños pequeños. En R. E. Tremblay, R. Barr, R. Peters \& M. Boivin (Eds.), Enciclopedia sobre el Desarrollo de la Primera Infancia. Recuperado de http://www.enciclopedia-infantes.com/documents/BiermanESPxp.pdf

Broidy, L. M., Nagin, D. S., Tremblay, R. E., Bates, J. E., Brame, B., Dodge, K. A., ... Vitaro, F. (2003). Developmental trajectories of childhood disruptive behaviors and adolescent delinquency: A six-site, crossnational study. Developmental Psychology, 39, 222-245.

Buehl, M. (2009). Exploring teachers' beliefs about teaching knowledge: Where does it come from? Does it change? The Journal of Experimental Education, 77(4), 367-407.

Calderhead, J. (1996). Teachers: Beliefs and knowledge. En D. Berliner \& R. Calfee (Eds.), Handbook of Educational Psychology (pp. 709-725). New York: Macmillan. 
Card, N., Stucky, B., Sawalani, G. \& Little, T. (2008). Direct and indirect aggression during childhood and adolescence: A meta-analytic review of gender differences, intercorrelations, and relations to maladjustment. Child Development, 79(5), 1185-1229.

Coie, J. K. \& Dodge, K. A. (1998). Aggression and antisocial behavior. En W. Damon (Ed. in Chief) \& N. Eisenberg (Vol. Ed.), Handbook of child psychology, 5th edition. Volume 3. Social, emotional, and personality development. New York: John Wiley \& Sons.

Corvalán, M. I. (2005). La realidad escolar cotidiana y la salud mental de los profesores. Enfoques Educacionales, 7(1), 69-79. Recuperado de http:// www.facso.uchile.cl/publicaciones/enfoques/09/Corvalan_N7_2005.pdf

Cerdá, A. (2004). Educación emocional y transformación de la escuela pública. En J. Ansión \& A. Villacorta (Eds.), Para comprender la escuela pública desde sus crisis y posibilidades (pp. 41-53). Lima: Fondo Editorial de la Pontificia Universidad Católica del Perú.

Cerezo F. (1997). Conductas agresivas en la edad escolar: aproximación teórica y metodológica. Propuesta de intervención. Madrid: Pirámide.

Chaux, E. (2003). Agresión reactiva, agresión instrumental y el ciclo de la violencia. Revista de Estudios Sociales, 15, 47-58.

De Rivera, J. (2003). Aggression, violence, evil, and peace. En I. Wiener. (Ed.), Handbook of Psychology (pp. 569-598). New Jersey: Wiley.

Discroll, H., Zinkivskay, A., Evans, K. \& Campbell, A. (2006). Gender differences in social representations of aggression: The phenomenological experience of differences in inhibitory control? British Journal of Psychology, 97, 139-153.

Dodge, K., Coie, J. \& Lynam, D. (2006). Aggression and antisocial behavior in youth. En W. Damon \& R. Lerner (Eds.), Handbook of Child Psychology (pp. 719-788). New Jersey: John Wiley.

Eisner, E. W. (2003). On the art and science of qualitative research in psychology. En P. M. Camic, J. E. Rhodes \& L. Yardley (Eds.), Qualitative research in psychology. Expanding perspectives in methodology and design ( $\mathrm{pp}$. 17-29). Washington D. C.: APA.

Furlong, M., Soliz, A., Simental, J. \& Greif, J. (2004). Bullying and abuse on school campuses. Encyclopedia of Applied Psychology, 1, 295-301. 
Gil-Verona, J., Pastor, J., De Paz, F., Barbosa, M., Macías, J., Maniega, M., ... Picornell, I. (2002). Psicobiología de las conductas agresivas. Anales de Psicología, 18(2), 293-303.

Gómez, V., Santa Cruz, J. \& Thomsen, P. (2007). En busca del cambio conceptual del profesor en el contexto de la formación permanente. REICE - Revista Electrónica Iberoamericana sobre Calidad, Eficacia y Cambio en Educación, 5(5e), 27-31. Recuperado de http://www.rinace.net/arts/vol5num5e/art3.pdf

Goetz, A. (2010). The evolutionary psychology of violence. Psicothema, 22(1), 15-21. Recuperado de http://web.ebscohost.com/ehost/pdf? vid=12\&hid=3\&sid=48b544b3-a693-49c4-83fb-9fa42cb0e50a\%40 sessionmgr13

Haney, J. \& Mcarthur, J. (2002). Four case studies of prospective teachers' beliefs concerning constructivist practice. Science Education, 86(6), 783-802.

Kagan, D. M. (1992). Implications of research on teacher belief. Educational Psychologist, 27, 65-90.

Kardash, C. A. M. \& Howell, K. L. (2000). Effects of epistemological beliefs and topic-specific beliefs on undergraduates' cognitive and strategic processing of dual-positional text. Journal of Educational Psychology, 92(3), 524-535.

Keenan, K. (2009). Desarrollo y socialización de la agresión durante los primeros cinco años de vida. En R. E. Tremblay, R. Barr, R. Peters \& M. Boivin (Eds.), Enciclopedia sobre el Desarrollo de la Primera Infancia. Recuperado de http://www.enciclopedia-infantes.com/documents/KeenanESPxp.pdf

Kyriacou, C. (2001). Teacher stress: Directions for future research. Educational Review, 53(1), 27-35.

Leal, F. (2005). Efecto de la formación docente inicial en las creencias epistemológicas. Revista Iberoamericana de Educación, 34(5). Recuperado de http://www.rieoei.org/deloslectores/803Leal.PDF

Lorenz, K. (1976). Sobre la agresión: el pretendido mal. México: Siglo XXI.

Macotela, S., Flores, R. \& Seda, I. (2001). Revista Iberoamericana de Educación OEI. Recuperado de http://www.rieoei.org/deloslectores/106Macotela.PDF

Magendzo, A. \& Toledo, M. (2008). Intimidación (bullying) en la escuela: investigaciones sobre clima y rendimiento escolar. En UNESCO, Organización de las Naciones Unidas para la Educación, la Ciencia y la Cultura \& Ministerio 
de Educación y Ciencia, España, II Jornadas de Cooperación con Iberoamérica sobre Educación en Cultura de Paz. Santiago de Chile: Salesianos impresores.

Malvaceda, E. (2009). Análisis psicosocial de la violencia, entre el conflicto y el desarrollo social. Cuadernos de Difusión, 14(16), 121-130. Recuperado de http://www.esan.edu.pe/publicaciones/cuadernos-de-difusion/26/ Malvaceda.pdf

Manson, J. \& Wrangham, R. W. (1991). Intergroup aggression in chimpanzees and humans. Current Anthropology, 32(4), 369-390.

Marcelo, C. (2005). La investigación sobre el conocimiento de los profesores y el proceso de aprender a enseñar. En G. Perafán \& A. Adúriz (Eds.), Pensamiento y conocimiento de los profesores. Debates y perspectivas internacionales. Bogotá: Universidad Pedagógica Nacional.

Ministerio de Educación del Perú. (2006). Comprendiendo la escuela desde su realidad cotidiana: estudio cualitativo en cinco escuelas estatales de Lima. Recuperado de http://www2.minedu.gob.pe/umc/admin/images/ cualitativo/estudio_cualitativo.pdf

Murray-Close, D. \& Ostrov, J. (2009). A longitudinal study of forms and functions of aggressive behavior in early childhood. Child Development, $80(3), 828-842$.

Nagin, D. S. \& Tremblay, R. E. (1999). Trajectories of boys' physical aggression, opposition, and hyperactivity on the path to physically violent and nonviolent juvenile delinquency. Child Development, 70, 1181-1196.

Nelson, R. J. \& Trainor, B. C. (2007). Neural mechanisms of aggression. Neuroscience, $8,536-546$.

Olafson, L. \& Schraw, G. (2006). Teachers' beliefs and practices within and across domains. International Journal of Educational Research, 45, 71-84.

Ortega, R. \& Mora-Merchán, J. A. (2000). Violencia escolar: mito o realidad. Sevilla: Mergablum.

Pajares, F. (1992). Teachers' beliefs and educational research: Cleaning up a messy construct. Review of Educational Research, 62(3), 305- 332.

Pepler, D. (2009). Apoyo a niños pequeños y sus familias para reducir la agresión. En R. E. Tremblay, R. Barr, R. Peters \& M. Boivin (Eds.), Enciclopedia sobre el Desarrollo de la Primera Infancia. Recuperado de http://www. enciclopedia-infantes.com/documents/PeplerESPxp.pdf 
Perlman, D. (1985). Psicología Social. México: Interamericana.

Plomin, R., Nitz, K. \& Rowe, D. (1990). Behavioral Genetics and Aggressive Behavior in Childhood. En S. Miller \& M. Lewis (Eds.), Handbook of Developmental Psychopathology (pp. 119-133). New York: Plenum.

Postigo, S., González, R., Mateu, C., Ferrero, J. \& Martorell, C. (2009). Diferencias conductuales según género en convivencia escolar. Psicothema, 21(3), 453-458.

Raine, A. (2002). Annotation: The role of prefrontal deficits, low autonomic arousal, and early health factors in the development of antisocial and aggressive behavior in children. Journal of Child Psychology and Psychiatry, 43(4), 417-434.

Real Academia Española (s. f.). Diccionario de la Lengua Española. Vigésima segunda edición. Recuperado de http://www.rae.es/rae.html

Roa, M., Del Barrio, M. \& Carrasco, M. (2004). Comparación de la agresión infantil en dos grupos culturales. Revista de Psicología, 22(1), 29-43.

Rodrigo, M., Rodríguez, A. \& Marrero, J. (1993). Las teorías implícitas: una aproximación al conocimiento cotidiano. Madrid: Visor.

Rokeach, M. (1968). Beliefs, attitudes, and values. San Francisco: Jossey-Bass.

Spilt, J. L., Koomen, H., Thijs, J. T., Stoel, R. D. \& Van der Leij, A. (2010). Teachers' assessment of antisocial behavior in kindergarten: Physical aggression and measurement bias across gender. Journal of Psychoeducational Assessment, 28(2), 129-138.

Tremblay, R. E. (2004). Decade of behavior distinguished lecture: Development of physical aggression during infancy. Infant Mental Health Journal, 25, 399-407.

Tremblay, R. E., Gervais, J. \& Petitclerc, A. (2008). Prevenir la violencia a través del aprendizaje de la primera infancia. Recuperado de http:// www.excellence-earlychildhood.ca/documents/Tremblay_ReporteAgresion_SP.pdf

Tremblay, R. E., Nagin, D. S., Séguin, J. R., Zoccolillo, M., Zelazo, P. D., Boivin, M., ... Japel, C. (2004). Physical aggression during early childhood: Trajectories and predictors. Pediatrics, 114(1), 43-50.

Tremblay, R. E. (2000). The development of aggressive behavior during childhood: What have we learned in the past century? International Journal of Behavioral Development, 24, 129-141. 
Tremblay, R. E., Japel, C., Pérusse, D., McDuff, P., Boivin, M., Zoccolillo, M. \& Montplaisir, J. (1999). The search for the age of "onset" of physical aggression: Rousseau and Bandura revisited. Criminal Behavior and Mental Health, 9, 8-23.

Tsouloupas, C., Carson, R., Matthews, R., Grawitch, M. \& Barber, L. (2010). Exploring the association between teachers' perceived student misbehaviour and emotional exhaustion: The importance of teacher efficacy beliefs and emotion regulation. Educational Psychology, 30(2), 173-189.

Underwood, M., Beron K., Gentsch, J., Galperin, M. \& Risser, S. (2008). Family correlates of children's social and physical aggression with peers: Negative interparental conflict strategies and parenting styles. International Journal of Behavioral Development, 32(6), 549-562.

UNESCO. (2005). Condiciones de trabajo y salud docente. Estudios de casos en Argentina, Chile, Ecuador, México, Perú y Uruguay. Santiago de Chile: UNESCO-OREALC.

Vitaro, F., Brendgen, M., Larose, S. \& Tremblay, R. E. (2005). Kindergarten disruptive behaviors, protective factors, and educational achievement by early adulthood. Journal of Educational Psychology, 97(4), 617-629.

Walker, P. L. (2001). A bioarchaeological perspective on the history of violence. Annual Review of Psychology, 30, 573-596.

Woollfolk, A., Davis, H. \& Pape, S. (2006). Teacher knowledge and beliefs. En P. Alexander \& P. Winne (Eds.), Handbook of Educational Psychology (pp. 715-737). Mahwah, NJ: Lawrence Erlbaum Associates.

Woolfolk, A. (1999). Psicología Educativa. México, D. F.: Prentice Hall.

Young, E., Boye, A. \& Nelson, D. (2006). Relational aggression: Understanding, identifying and responding in schools. Psychology in the Schools, 43(3), 297-312. 